\title{
Relic neutrino asymmetry, CMB and large-scale structure
}

\author{
Sergio Pastor* a and Julien Lesgourgues ${ }^{\dagger}$ a \\ ${ }^{a}$ SISSA-ISAS and INFN, Sezione di Trieste \\ Via Beirut 2-4, I-34013 Trieste, Italy
}

\begin{abstract}
We consider some consequences of the presence of a cosmological lepton asymmetry in the form of neutrinos. A relic neutrino degeneracy enhances the contribution of massive neutrinos to the present energy density of the Universe, and modifies the power spectrum of radiation and matter. Comparing with current observations of cosmic microwave background anisotropies and large scale structure, we derive some constraints on the relic neutrino degeneracy and on the spectral index in the case of a flat Universe with a cosmological constant.
\end{abstract}

\section{Introduction}

It is generally assumed that our Universe contains an approximately equal amount of leptons and antileptons. The lepton asymmetry would be of the same order as the baryon asymmetry, which is very small as required by Big Bang Nucleosynthesis (BBN) considerations. The existence of a large lepton asymmetry is restricted to be in the form of neutrinos from the requirement of universal electric neutrality, and the possibility of a large neutrino asymmetry is still open. From a particle physics point of view, a lepton asymmetry can be generated by an Affleck-Dine mechanism [1] without producing a large baryon asymmetry (see ref. [2] for a recent model), or even by active-sterile neutrino oscillations after the electroweak phase transition [3].

We have studied some cosmological implica-

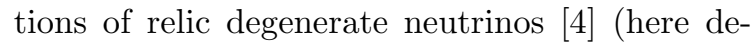
generate refers to neutrino-antineutrino asymmetry, not to mass degeneracy). We do not consider any specific model for generating such an asymmetry, and just assume that it was created well before neutrinos decouple from the rest of the plasma. An asymmetry of order one or larger can have crucial effects on the global evolution of the Universe. Among other effects, it changes the decoupling temperature of neutrinos, the primordial production of light elements at BBN, the time

\footnotetext{
*Speaker, e-mail: pastor@sissa.it

${ }^{\dagger}$ E-mail: lesgour@sissa.it. Work supported by INFN and by the TMR network grant ERBFMRXCT960090.
}

of equality between radiation and matter, or the contribution of relic neutrinos to the present energy density of the Universe. The latter changes affect the evolution of perturbations in the Universe. We focus on the anisotropies of the Cosmic Microwave Background (CMB), and on the distribution of Large Scale Structure (LSS). We calculate the power spectrum of both quantities, in the case of massless degenerate neutrinos, and also for neutrinos with a mass of $0.07 \mathrm{eV}$, as suggested to explain the experimental evidence of atmospheric neutrino oscillations at Super-Kamiokande [5].

The effect of neutrino degeneracy on the LSS power spectrum was studied in ref. [6], as a way of improving the agreement with observations of mixed dark matter models with $\mathrm{eV}$ neutrinos, in the case of high values of the Hubble parameter. Adams \& Sarkar [7] calculated the CMB anisotropies and the matter power spectrum, and compared them with observations in the $\Omega_{\Lambda}=0$ case for massless degenerate neutrinos. More recently, Kinney \& Riotto [8] also calculated the CMB anisotropies for massless degenerate neutrinos in the $\Omega_{\Lambda}=0.7$ case.

\section{Energy density of massive degenerate neutrinos}

The energy density of one species of massive degenerate neutrinos and antineutrinos, described by the distribution functions $f_{\nu}$ and $f_{\bar{\nu}}$, is (we use $\hbar=c=k_{B}=1$ units)

$\rho_{\nu}+\rho_{\bar{\nu}}=\int_{0}^{\infty} \frac{d p}{2 \pi^{2}} p^{2} \sqrt{p^{2}+m_{\nu}^{2}}\left(f_{\nu}(p)+f_{\bar{\nu}}(p)\right)$ 
valid at any moment. Here $p$ is the magnitude of the 3-momentum and $m_{\nu}$ is the neutrino mass.

When the early Universe was hot enough, the neutrinos were in equilibrium with the rest of the plasma via the weak interactions. In that case the distribution functions $f_{\nu}$ and $f_{\bar{\nu}}$ changed with the Universe expansion, keeping the form of a FermiDirac distribution,

$$
f_{\nu, \bar{\nu}}(p)=\frac{1}{\exp \left(\frac{p}{T_{\nu}} \mp \frac{\mu}{T_{\nu}}\right)+1}
$$

Here $\mu$ is the neutrino chemical potential, which is nonzero if a neutrino-antineutrino asymmetry has been previously produced. Later the neutrinos decoupled when they were still relativistic, and from that moment the neutrino momenta just changed according to the cosmological redshift. If $a$ is the expansion factor, the neutrino momentum decreases keeping ap constant. At the same time the neutrino degeneracy parameter $\xi \equiv \mu / T_{\nu}$ is conserved, with a value equal to that at the moment of decoupling. Therefore one can still calculate the energy density of neutrinos now from eq. (11) and eq. (2), replacing $\mu / T_{\nu}$ by $\xi$ and $p / T_{\nu}$ by $p /\left(y_{\nu} T_{0}\right)$, where $T_{0} \simeq 2.726 \mathrm{~K}$ and $y_{\nu}$ is the present ratio of neutrino and photon temperatures, which is not unity because once decoupled the neutrinos did not share the entropy transfer to photons from the successive particle annihilations that occurred in the early Universe.

In the presence of a significant neutrino degeneracy $\xi$ the decoupling temperature $T(\xi)$ is higher than in the standard case, [9, 10]. The reaction rate $\Gamma$ of the weak processes, that keep the neutrinos in equilibrium with the other species, is reduced because some of the initial or final neutrino states will be occupied. The authors of ref. [10] found that the neutrino decoupling temperature is $T_{d e c}(\xi) \approx 0.2 \xi^{2 / 3} \exp (\xi / 3) \mathrm{MeV}$ (for $\nu_{\mu}$ or $\left.\nu_{\tau}\right)$. Therefore if $\xi$ is large enough, the degenerate neutrinos decouple before the temperature of the Universe drops below the different mass thresholds, and are not heated by the particleantiparticle annihilations, reducing the ratio of neutrino and photon temperatures with respect to the standard value $y_{\nu}=(4 / 11)^{1 / 3}$.
The present contribution of these degenerate neutrinos to the energy density of the Universe can be parametrized as $\rho_{\nu}=10^{4} h^{2} \Omega_{\nu} \mathrm{eV} \mathrm{cm}^{-3}$, where $\Omega_{\nu}$ is the neutrino energy density in units of the critical density $\rho_{c}=3 H^{2} M_{P}^{2} / 8 \pi, M_{P}=$ $1.22 \times 10^{19} \mathrm{GeV}$ is the Planck mass and $H=100 \mathrm{~h}$ $\mathrm{Km} \mathrm{s}^{-1} \mathrm{Mpc}^{-1}$ is the Hubble parameter. The value of $\rho_{\nu}$ can be calculated as a function of the neutrino mass and the neutrino degeneracy $\xi$, or equivalently the present neutrino asymmetry $L_{\nu}$ defined as the following ratio of number densities

$L_{\nu} \equiv \frac{n_{\nu}-n_{\bar{\nu}}}{n_{\gamma}}=\frac{1}{12 \zeta(3)} y_{\nu}^{3}\left[\xi^{3}+\pi^{2} \xi\right]$

We show ${ }^{3}$ in figure 1 the contours in the $\left(m_{\nu}, L_{\nu}\right)$ plane that correspond to some particular values of $h^{2} \Omega_{\nu}$. In the limit of small degeneracy (vertical lines) one recovers the well-known bound on the neutrino mass $m_{\nu} \lesssim 46 \mathrm{eV}$ for $h^{2} \Omega_{\nu}=0.5$. On the other hand, for very light neutrinos the horizontal lines set a maximum value on the neutrino degeneracy, that would correspond to a present neutrino chemical potential $\mu_{0} \lesssim 7.4 \times 10^{-3} \mathrm{eV}$, also for $h^{2} \Omega_{\nu}=0.5$. In the intermediate region of the figures the neutrino energy density is $\rho_{\nu} \simeq m_{\nu} n_{\nu}(\xi)$ and the contours follow roughly the relation $L_{\nu}\left(m_{\nu} / \mathrm{eV}\right) \simeq 24.2 h^{2} \Omega_{\nu}$.

A similar calculation has been recently performed in reference [11]. Note however that the ratio of neutrino and photon temperatures was not properly taken into account for large $\xi$.

The presence of a neutrino degeneracy can modify the outcome of BBN (for a review see [12]). First a larger neutrino energy density increases the expansion rate of the Universe, thus enhancing the primordial abundance of ${ }^{4} \mathrm{He}$. This is valid for a nonzero $\xi$ of any neutrino flavor. In addition if the degenerate neutrinos are of electron type, they have a direct influence over the weak processes that interconvert neutrons and protons. This last effect depends on the sign of $\xi_{\nu_{e}}$, and one gets $-0.06 \lesssim \xi_{\nu_{e}} \lesssim 1.1$ [10], while a sufficiently long matter dominated epoch requires $\left|\xi_{\nu_{\mu}, \nu_{\tau}}\right| \lesssim 6.9$ [10]. This estimate agrees with our analysis in section 1 and places a limit shown by

\footnotetext{
${ }^{3}$ Here we assume $\xi>0$, but the results are also valid for $\xi<0$ provided that $\xi$ and $L_{\nu}$ are understood as moduli.
} 
the horizontal line in figure 1 in the case of degenerate $\nu_{\mu}$ or $\nu_{\tau}$.

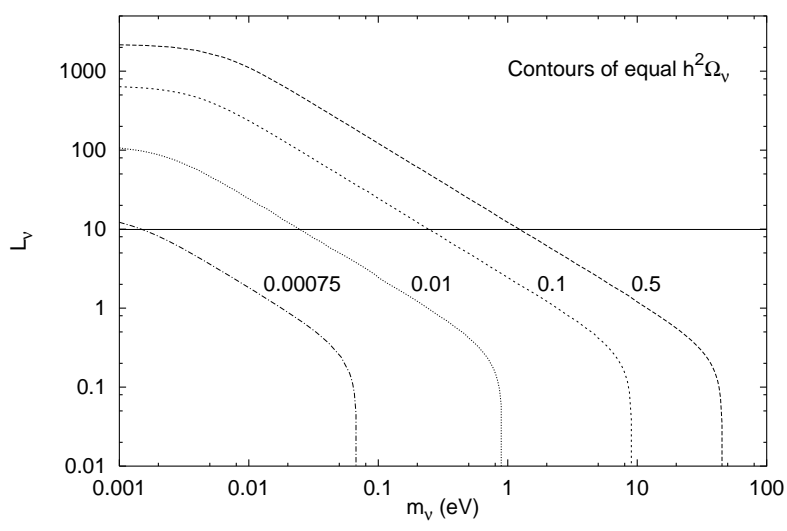

Figure 1. Present energy density of massive degenerate neutrinos as a function of the neutrino asymmetry.

\section{Effects on the power spectra}

We compute the power spectra of CMB anisotropies and LSS using the Boltzmann code cmbfast by Seljak \& Zaldarriaga [13], adapted to the case of one family of degenerate neutrinos $(\nu$, $\bar{\nu})$, with mass $m_{\nu}$ and degeneracy parameter $\xi$. Our modifications to the code are reviewed and explained in 四.

The effect of $\xi$ and $m_{\nu}$ on the CMB anisotropy spectrum can be seen in figure 2. We choose a set of cosmological parameters $\left(h=0.65, \Omega_{b}=0.05\right.$, $\Omega_{\Lambda}=0.70, \Omega_{C D M}=1-\Omega_{b}-\Omega_{\nu}-\Omega_{\Lambda}, Q_{r m s-p s}=$ $18 \mu \mathrm{K}$, flat primordial spectrum, no reionization, no tensor contribution), and we vary $\xi$ from 0 to 5 , both in the case of massless degenerate neutrinos and degenerate neutrinos with $m_{\nu}=0.07 \mathrm{eV}$.

Let us first comment the massless case. The main effect of $\xi$ is to boost the amplitude of the first peakf. Indeed, increasing the energy density of radiation delays matter-radiation equality,

\footnotetext{
${ }^{4}$ In fact, this is not true for very large values of $\xi$, where recombination can take place still at the end of radiation domination, and anisotropies are suppressed. However in such a case the location of the first peak is $l \gtrsim 450$, and the matter power spectrum is strongly suppressed.
}

which is known to boost the acoustic peaks, and to shift them to higher multipoles, by a factor $\left(\left(1+a_{e q} / a_{*}\right)^{1 / 2}-\left(a_{e q} / a_{*}\right)^{1 / 2}\right)^{-1}\left(a_{e q}\right.$ increases with $\xi$, while the recombination scale factor $a_{*}$ is almost independent of the radiation energy density). Secondary peaks are then more affected by diffusion damping at large $l$, and their amplitude can decrease with $\xi$.

In the case of degenerate neutrinos with $m_{\nu}=$ $0.07 \mathrm{eV}$, the results are quite similar in first approximation. Indeed, the effects described previously depend on the energy density of neutrinos at equality. At that time, they are still relativistic, and identical to massless neutrinos with equal degeneracy parameter. However, with a large degeneracy, $\Omega_{\nu}$ today becomes significant: for $\xi=5$, one has $\Omega_{\nu}=0.028$, i.e. the same order of magnitude as $\Omega_{b}$. Since we are studying flat models, $\Omega_{\nu}$ must be compensated by less baryons, cold dark matter $(\mathrm{CDM})$ or $\Omega_{\Lambda}$. In our example, $\Omega_{b}$ and $\Omega_{\Lambda}$ are fixed, while $\Omega_{C D M}$ slightly decreases. This explains the small enhancement of the first peak compared to the massless case $(3.4 \%$ for $\xi=5)$. Even if this effect is indirect, it is nevertheless detectable in principle, possibly by future satellite missions MAP and Planck (even if one does not impose the flatness condition, the effect of $\Omega_{\nu}$ will be visible through a modification of the curvature).

We also plot in figure 2 the power spectrum $P(k)$, normalized on large scales to COBE. The effect of both parameters $\xi$ and $m_{\nu}$ is now to suppress the power on small scales. Indeed, increasing $\xi$ postpones matter-radiation equality, allowing less growth for fluctuations crossing the Hubble radius during radiation domination. Adding a small mass affects the recent evolution of fluctuations, and has now a direct effect: when the degenerate neutrinos become non-relativistic, their free-streaming suppresses the growth of fluctuations for scales within the Hubble radius. This effect, already known for non-degenerate neutrinos 14, is enhanced in the presence of a neutrino degeneracy, since the average neutrino momentum is shifted to larger values.

Our results for massless degenerate neutrinos can be compared with those of previous works. We found the same effect of $\xi$ on the CMB for 

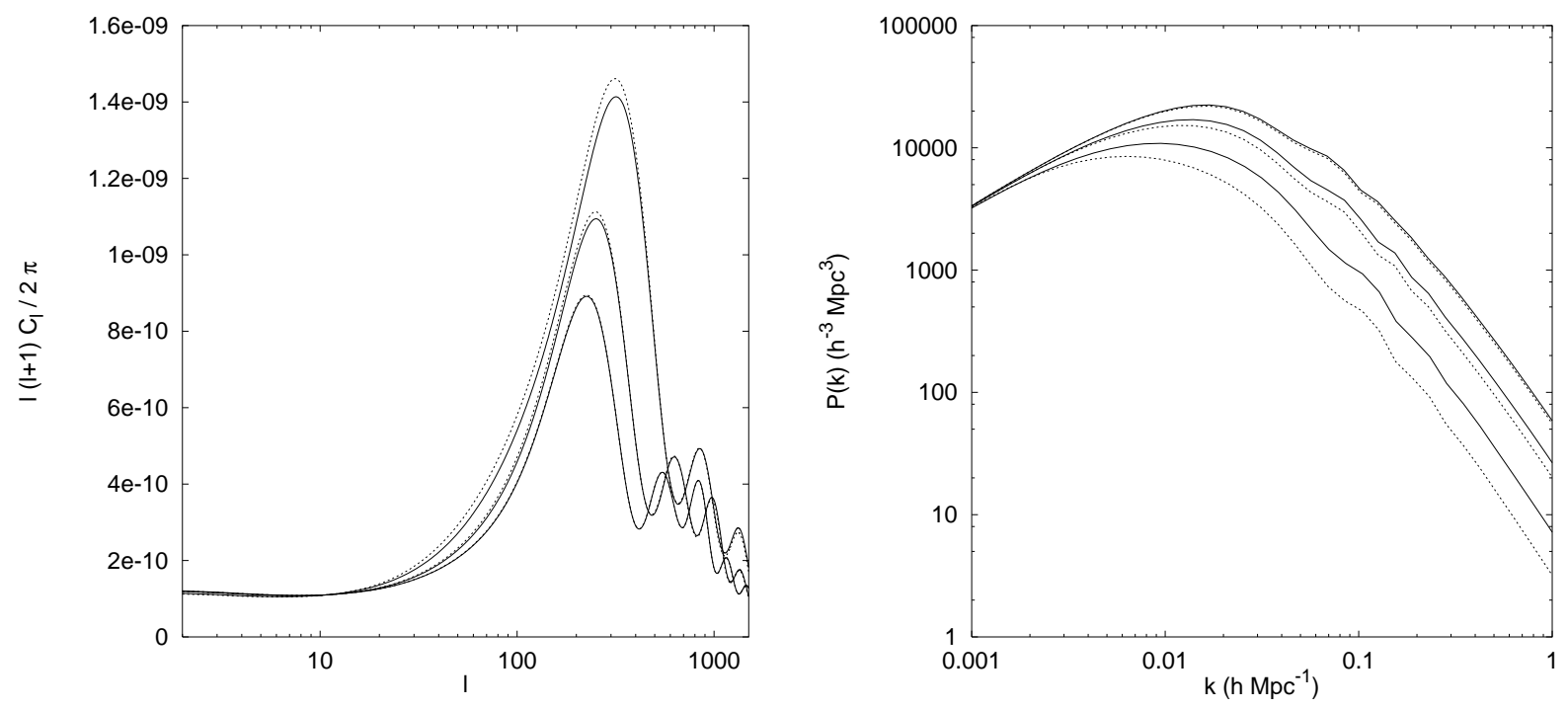

Figure 2. CMB anisotropy and matter power spectra for different models with one family of massless (solid lines) and $m_{\nu}=0.07 \mathrm{eV}$ (dashed lines) degenerate neutrinos. From bottom to top (from top to bottom for $P(k)), \xi=0,3,5$. Cosmological parameters are fixed as described in the text.

$\Omega_{\Lambda}=0$ as in [7], while the revised results in 8] also agree with our calculations for $\Omega_{\Lambda}=0.7$.

\section{Comparison with observations}

Since the degeneracy increases dramatically the amplitude of the first CMB peak, we expect large $\xi$ values to be favored in the case of cosmological models known to predict systematically a low peak (unless a large blue tilt is invoked, which puts severe constraints on inflation). Our goal here is not to explore systematically all possibilities, but to briefly illustrate how $\xi$ can be constrained by current observations for flat models with different values of $\Omega_{\Lambda}$. Recent results from supernovae, combined with CMB constraints, favor flat models with $\Omega_{\Lambda} \sim 0.6-0.7$.

We choose a model with $h=0.65, \Omega_{b}=0.05$, $Q_{r m s-p s}=18 \mu \mathrm{K}$, no reionization and no tensor contribution, and look for the allowed window in the space of free parameters $\left(\Omega_{\Lambda}, \xi, n\right)$. The allowed window is defined as the intersection of regions preferred at the $95 \%$ confidence level by four independent experimental tests, based on $\sigma_{8}$ estimation, Stromlo-APM redshift survey, bulk ve- locity reconstruction, and CMB anisotropy measurements. Details concerning these tests can be found in $[$ 汪.

We plot in figure 3 the LSS and CMB allowed regions in $(\xi, n)$ parameter space, for $\Omega_{\Lambda}=0$ and 0.6. In the case of degenerate neutrinos with $m_{\nu}=0.07 \mathrm{eV}$, the LSS regions are slightly shifted at large $\xi$, since, as we saw, the effect of $\xi$ is enhanced (dotted lines on the figure). The CMB regions do not show this distinction, given the smallness of the effect and the imprecision of the data. One can immediately see that LSS and CMB constraints on $n$ are shifted in opposite direction with $\xi$ : indeed, the effects of $\xi$ and $n$ both produce a higher CMB peak, while to a certain extent they compensate each other in $P(k)$. So, for $\Omega_{\Lambda} \geq 0.7$, a case in which a power spectrum normalized to both COBE and $\sigma_{8}$ yields a too high peak 5 , a neutrino degeneracy can only make things worst, and we find no allowed window at all. In the other extreme case $\Omega_{\Lambda}=0$, it is well known that the amplitude required by $\sigma_{8}$ and the

\footnotetext{
${ }^{5} \mathrm{At}$ least, for the values of the other cosmological parameters considered here. This situation can be easily improved, for instance, with $h=0.7$.
} 

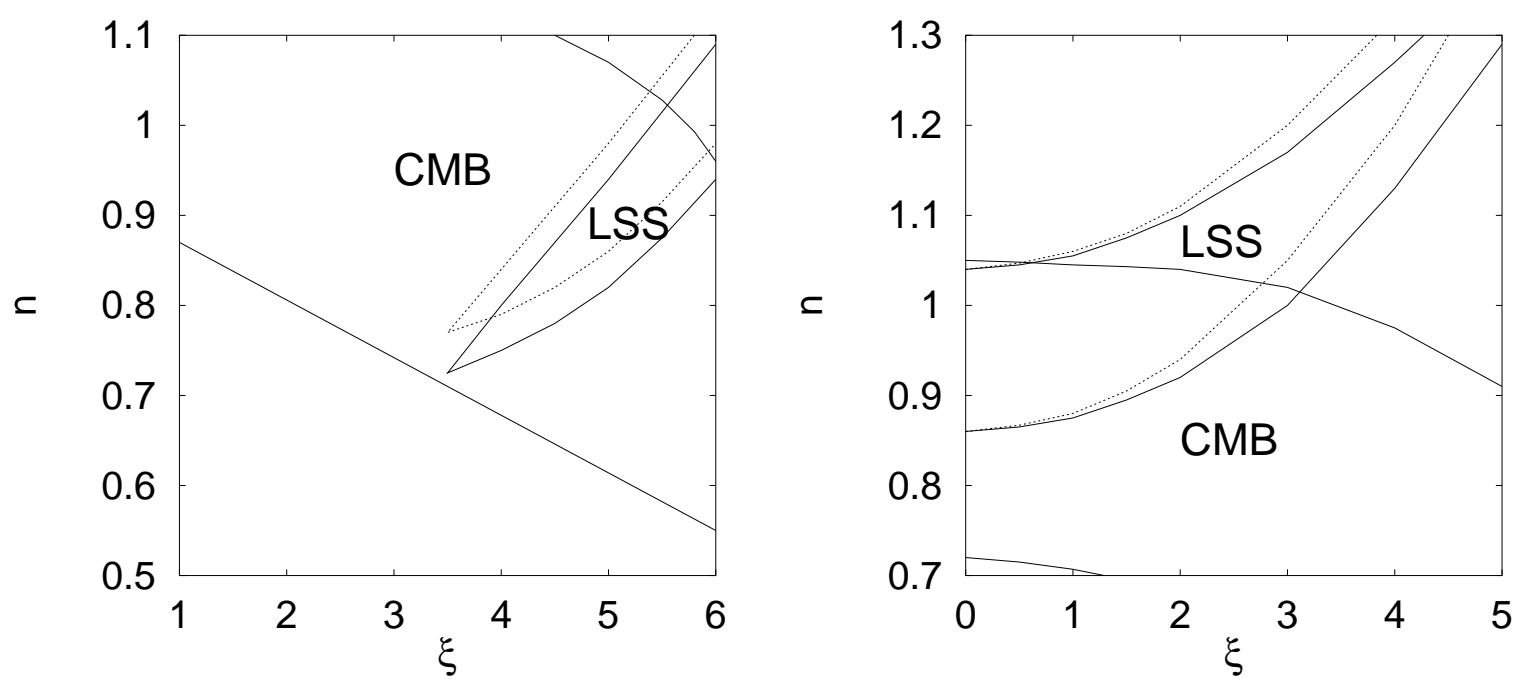

Figure 3. LSS and CMB constraints in $(\xi, n)$ space for $\Omega_{\Lambda}=0$ (left) and $\Omega_{\Lambda}=0.6$ (right). The underlying cosmological model is flat, with $h=0.65, \Omega_{b}=0.05, Q_{r m s-p s}=18 \mu \mathrm{K}$, no reionization, no tensor contribution. The allowed regions are those where the labels are. For LSS constraints, we can distinguish between degenerate neutrinos with $m_{\nu}=0$ (solid lines) and $m_{\nu}=0.07 \mathrm{eV}$ (dotted lines).

shape probed by redshift surveys favor different values of $n$. We find that the neutrino degeneracy can solve this problem with $\xi \gtrsim 3.5$, but the allowed window is cut at $\xi \simeq 6$ by $\mathrm{CMB}$ data, and we are left with an interesting region in which $\Omega_{0}=1$ models are viable. This result is consistent with [7]. However, current evidences for a low $\Omega_{0}$ Universe are independent of the constraints used here, so there are not many motivations at the moment to consider this window seriously. Finally, for $\Omega_{\Lambda}=0.5-0.6$, a good agreement is found up to $\xi \simeq 3$. This upper bound could marginally explain the generation of ultrahigh energy cosmic rays by the annihilation of high-energetic neutrinos on relic neutrinos with mass $m_{\nu}=0.07 \mathrm{eV}[15]$.

\section{REFERENCES}

1. I. Affleck and M. Dine, Nucl. Phys. B249 (1985) 361.

2. A. Casas, W.Y. Cheng and G. Gelmini, Nucl. Phys. B538 (1999) 297.

3. R. Foot, M.J. Thomson and R.R. Volkas, Phys. Rev. D53 (1996) 5349.
4. J. Lesgourgues and S. Pastor, preprint hep$\mathrm{ph} / 9904411$.

5. Y. Fukuda et al. [Super-Kamiokande Collaboration], Phys. Rev. Lett. 81 (1998) 1562.

6. G.B. Larsen and J. Madsen, Phys. Rev. D52 (1995) 4282.

7. J.A. Adams and S. Sarkar, preprint OUTP98-70P and talk presented at The Physics of Relic Neutrinos, Trieste, September 1998.

8. W.H. Kinney and A. Riotto, preprint hep$\mathrm{ph} / 9903459$.

9. K. Freese, E.W. Kolb and M.S. Turner, Phys. Rev. D27 (1983) 1689.

10. H. Kang and G. Steigman, Nucl. Phys. B372 (1992) 494.

11. P.B. Pal and K. Kar, Phys. Lett. B451 (1999) 136.

12. S. Sarkar, Rept. Prog. Phys. 59 (1996) 1493.

13. U. Seljak and M. Zaldarriaga, Astrophys. J. 469 (1996) 437.

14. W.T. Hu, D.J. Eisenstein and M. Tegmark, Phys. Rev. Lett. 80 (1998) 5255.

15. G. Gelmini and A. Kusenko, Phys. Rev. Lett. 82 (1999) 5202. 